\title{
Electron spin resonance detected by a superconducting qubit
}

\author{
Y. Kubo ${ }^{1}$, I. Diniz ${ }^{2}$, C. Grezes ${ }^{1}$, T. Umeda ${ }^{3}$, J. Isoya ${ }^{3}$, H. Sumiya ${ }^{4}$, T. Yamamoto ${ }^{5}$, H. Abe ${ }^{5}$, S. Onoda ${ }^{5}$, T. \\ Ohshima $^{5}$, V. Jacques ${ }^{6}$, A. Dréau ${ }^{6}$, J.-F. Roch ${ }^{6}$, A. Auffeves ${ }^{2}$, D. Vion ${ }^{1}$, D. Esteve ${ }^{1}$, and P. Bertet ${ }^{1}$ \\ ${ }^{1}$ Quantronics group, SPEC (CNRS URA 2464), CEA-Saclay, 91191 Gif-sur-Yvette, France \\ ${ }^{2}$ Institut Néel, CNRS, BP 166, 38042 Grenoble, France \\ 3 Research Center for Knowledge Communities, University of Tsukuba, Tsukuba 305-8550, Japan \\ ${ }^{4}$ Sumitomo Electric Industries Ltd., Itami 664-001, Japan \\ 5 Japan Atomic Energy Agency, Takasaki 370-1292, Japan and \\ ${ }^{6}$ LPQM (CNRS UMR 8537), ENS de Cachan, 94235 Cachan, France
}

(Dated: November 6, 2018)

\begin{abstract}
A new method for detecting the magnetic resonance of electronic spins at low temperature is demonstrated. It consists in measuring the signal emitted by the spins with a superconducting qubit that acts as a single-microwave-photon detector, resulting in an enhanced sensitivity. We implement this new type of electron-spin resonance spectroscopy using a hybrid quantum circuit in which a transmon qubit is coupled to a spin ensemble consisting of NV centers in diamond. With this setup we measure the NV center absorption spectrum at $30 \mathrm{mK}$ at an excitation level of $\sim 15 \mu_{B}$ out of an ensemble of $10^{11}$ spins.
\end{abstract}

\section{INTRODUCTION}

Electron spin resonance (ESR) spectroscopy at low temperatures is often complicated by the long spin-lattice energy relaxation time which can reach minutes at subkelvin temperatures. For continuous-wave (CW) ESR spectroscopy, this implies working at low powers to avoid saturating the spins; in pulsed ESR this imposes low repetition rates. In both cases a higher sensitivity for detecting the signal absorbed or emitted by the spins would be desirable. Recently, tools borrowed from superconducting quantum electronics have been applied to highsensitivity ESR spectroscopy. High-Q superconducting coplanar waveguide resonators have been used for CWESR at millikelvin temperatures with a cryogenic lownoise HEMT amplifier followed by homodyne detection [1 4], and for pulsed ESR at kelvin temperatures with a commercial ESR spectrometer [5], yielding promising results in terms of sensitivity. Here we go one step further and use an on-chip single microwave photon detector 6 based on a superconducting qubit [7] to realize a new type of high-sensitivity low-temperature ESR spectrometer.

The principle of our experiment is compared to more conventional ESR techniques in Fig. 1. In conventional ESR spectroscopy, a microwave pulse is applied to an ensemble of spins close to their resonance frequency $\omega_{s}$ through a low-Q cavity of frequency $\omega_{c}$ with which the spins are tuned in resonance $\left(\omega_{s}=\omega_{c}\right)$ by a magnetic field. After being excited by the pulse, the spins reradiate coherently part of the absorbed energy through the cavity into the detection waveguide, giving rise to a free induction decay (FID) signal measured by homodyne detection, which yields after Fourier transform the spin absorption spectrum 8]. What limits the sensitivity of a typical commercial $\mathrm{CW}$-spectrometer operating at $300 \mathrm{~K}$ to $\sim 10^{10}$ spins $/ \sqrt{\mathrm{Hz}}$ for a linewidth of $0.1 \mathrm{mT}$ and an integration time of $1 \mathrm{~s}$ is the overall noise temperature of the detection chain. In this work, we replace the detection chain by a superconducting qubit and its readout
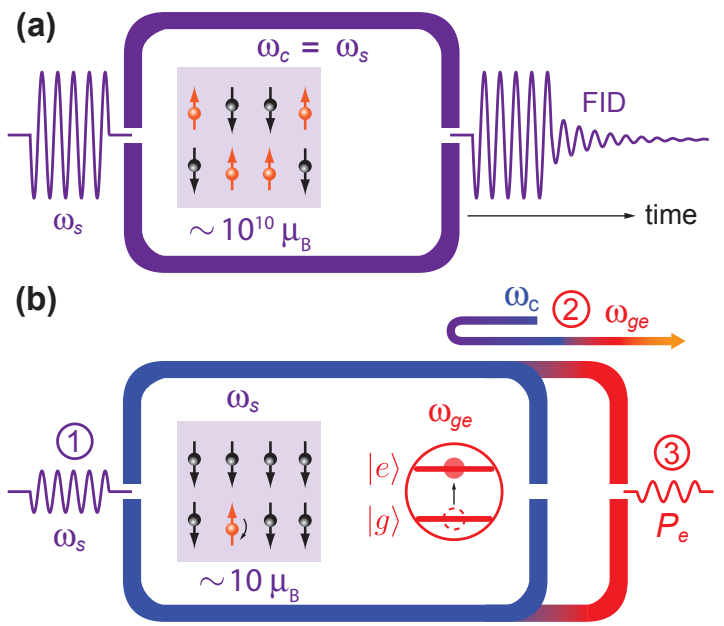

Figure 1. Comparison between conventional pulsed electron spin resonance (ESR) and qubit-detected ESR. a, Conventional ESR: A spin ensemble is placed in a cavity with frequency $\omega_{c}$ and driven with a microwave pulse resonant with the cavity. When the ESR frequency $\omega_{s}$ matches $\omega_{c}$, the spins absorb the microwave pulse, and emit immediately after a free-induction decay (FID) signal into the waveguide connected to the cavity. b, Qubit-detected ESR: The cavity is now frequency-tunable and embeds both the spin ensemble and a superconducting qubit with frequency $\omega_{g e}$. In a first step (1), the spins are probed by a spectroscopy pulse which excites them if its frequency matches $\omega_{s}$. In a second step (2), the cavity frequency is tuned to $\omega_{c}=\omega_{s}$, receives the FID signal from the spins, and is afterwards tuned to transfer this signal to the superconducting qubit at $\omega_{g e}$. Finally (3) the qubit excited state probability $P_{e}\left(\omega_{s}\right)$ is measured, mapping the spins absorption spectrum. Very low excitation powers can be used given the high sensitivity of the method.

circuitry. This results in an increased sensitivity since a superconducting qubit is a nearly ideal single microwave photon detector [6] at its resonance frequency $\omega_{g e}$. In order to transfer part of the excitation of the spins to 


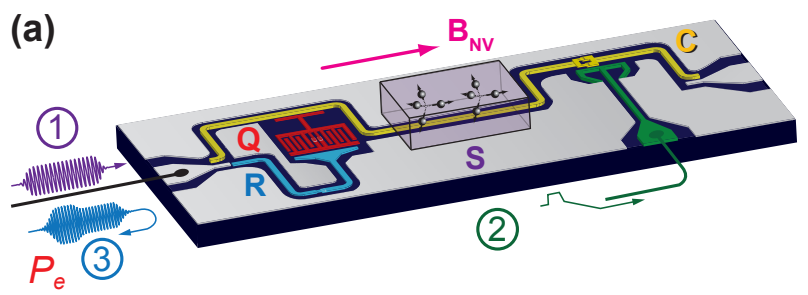

(b)
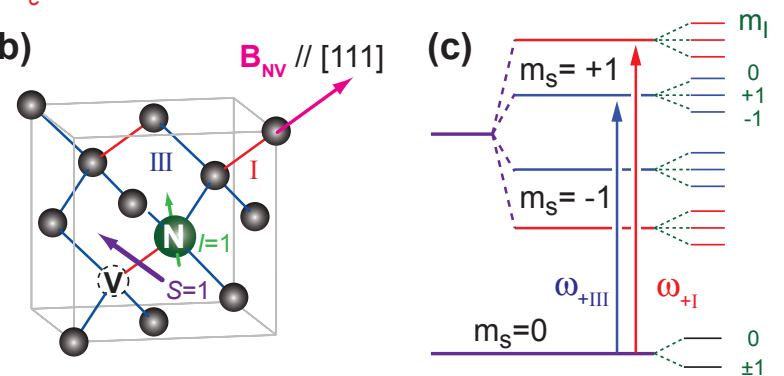

Figure 2. a, Sketch of the implementation of qubit-based ESR. The spin ensemble $S$ consists of NV centers in a diamond crystal. They are coupled to the frequency-tunable coplanar waveguide resonator $C$ used as the ESR cavity. $C$ also embeds the ESR-detector qubit $Q$, a superconducting qubit of the transmon type whose state can be readout with another resonator $R$. Microwave pulses for spin spectroscopy as well as for qubit readout are sent via an input port coupled both to $C$ and to $R$. $\mathbf{b}$ and $\mathbf{c}$, Sketch and energy levels of NV centers in diamond. In our setup a DC magnetic field $\mathbf{B}_{N V}$ is applied along the $[1,1,1]$ direction, resulting in different Zeeman splittings for centers having the $N-V$ axis parallel $\mathbf{B}_{N V}$ (ensemble $I$, in red) and those having their axis along the three other $\langle 1,1,1\rangle$ axes (ensemble $I I I$, in blue). The ESR frequencies $\omega_{ \pm I, I I I}$ are further split in three resonance lines due to the hyperfine interaction with the spin-1 ${ }^{14} N$ nuclear spin [11.

the superconducting qubit, the ESR resonator is made frequency-tunable and with a high quality factor. Spectroscopy is performed by first exciting the spins with a weak microwave pulse, collecting the radiated FID signal with the resonator tuned at $\omega_{s}$, then transferring this signal to the qubit at $\omega_{g e}$, and measuring its final state. Repeating this experimental sequence yields the probability $P_{e}$ to find the qubit in its excited state, which reproduces the spin absorption spectrum. The sensitivity of such an ESR spectrometer is set by the efficiency at which signal photons can be transferred from the spins to the resonator, then to the qubit, and by the fidelity with which the qubit state can be measured.

\section{DEVICE AND EXPERIMENTAL SETUP}

We implement this method using a recently reported hybrid quantum circuit [9] that includes an ensemble of electronic spins magnetically coupled to a superconducting resonator, itself electrically coupled to a superconducting qubit, as sketched in Fig. 2a. The spins are negatively-charged nitrogen-vacancy (NV) color centers in diamond, whose structure and energy levels are summarized in Fig $2 \mathrm{~b}$ and c. The ground state of NV centers has a spin one with splitting $\omega_{ \pm} / 2 \pi \simeq 2.88 \mathrm{GHz}$ between states $m_{S}=0$ and $m_{S}= \pm 1$ at zero magnetic field [10]. Each of the two $m_{S}=0$ to $m_{S}= \pm 1$ transitions is further splitted into three peaks separated by $2.2 \mathrm{MHz}$ due to the hyperfine (HF) coupling to the ${ }^{14} \mathrm{~N}$ nuclear spin [11]. In the experiment a static magnetic field $B_{N V}=1.1 \mathrm{mT}[12$ ] is applied along the $[1,1,1]$ crystallographic axis to lift the degeneracy between states $m_{S}= \pm 1$. Centers having their $N-V$ axis along $[1,1,1]$ (called in the following ensemble $I$ ) undergo a different Zeeman shift from those along the three other $\langle 1,1,1\rangle$ axes (ensemble $I I I)$, resulting in two different ESR frequencies $\omega_{+\mathrm{I}} / 2 \pi=2.91 \mathrm{GHz}$ and $\omega_{+\mathrm{III}} / 2 \pi=2.89 \mathrm{GHz}$ for the $m_{S}=0$ to $m_{S}=+1$ transition on which we will exclusively focus in the following.

The diamond crystal used is of the High-Pressure HighTemperature (HPHT) Ib type and has a NV center concentration of $\sim 3 \mathrm{ppm}$ and a residual nitrogen concentration of $\sim 30 \mathrm{ppm}$. In our setup, it is glued on top of the ESR cavity $C$, a coplanar waveguide superconducting resonator [13] of quality factor $Q \sim 10^{4}$ made of a niobium thin-film sputtered on a silicon substrate. The spin ensemble $S$ detected in the experiment consists of the $\sim 10^{11} \mathrm{NV}$ centers that lie within the mode volume of $C$, thus within a few microns of the diamond surface. The cavity frequency $\omega_{c}(\Phi)$ can be tuned on a nanosecond timescale by a flux $\Phi$ applied through the loop of a superconducting quantum interference device (SQUID) inserted into $C$ [1, 14 16]. The superconducting qubit is a Cooper-pair box of the transmon type [17, 18] with resonance frequency $\omega_{g e}$ between its ground state $|g\rangle$ and excited state $|e\rangle$. It is coupled to an additional resonator $R$ which is nonlinear and used to read-out the qubit state. As explained in details in Ref. [19, this readout is performed in a single shot by measuring the phase of a microwave pulse reflected on $R$. This phase takes two different values depending on the qubit state, and repeating $\sim 10^{4}$ times the same experimental sequence yields the qubit excited state probability $P_{e}$.

\section{SINGLE PHOTON STORAGE AND RETRIEVAL}

As reported in an earlier work [9] (see also [21]), it is possible with this circuit to coherently exchange a single quantum of excitation between the spin ensemble $S$ and the qubit $Q$ via the tunable cavity $C$. To demonstrate that, the qubit is prepared in $|e\rangle$; its excitation is transferred to the cavity by sweeping adiabatically $\omega_{c}(\Phi)$ through $\omega_{g e}$, which is then tuned suddently in resonance with the spins at $\omega_{K}$ (where $K=+I,+I I I$ ) for some interaction time $\tau$. The excitation left in the cavity is finally transferred back into the qubit, which is then readout. As shown in Fig. 3, the resulting qubit excited state probability $P_{e}(\tau)$ is found to oscillate, revealing the 


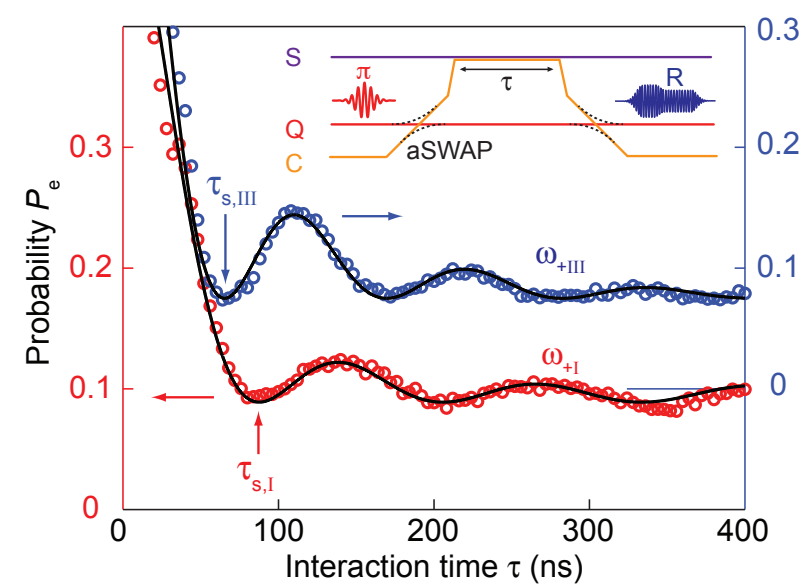

Figure 3. Single photon transfer between qubit and spins [9]. (inset): Pulse sequence used to excite the qubit in state $|e\rangle$ and transfer its excitation to $S$ via $C$ back and forth. (panel): Swap oscillations for the two spin frequencies $\omega_{+\mathrm{I}}$ and $\omega_{+\mathrm{III}}$. Swap times $\tau_{\mathrm{S}, \mathrm{I}}$ and $\tau_{\mathrm{s}, \mathrm{III}}$ are indicated by arrows. Note that the two curves are shifted vertically for clarity with corresponding scales on left (for ensemble $I$ ) and right axes (ensemble $I I I$ ).

conversion of a single microwave photon into an elementary collective excitation of the spin ensemble. For welldefined interaction times $\tau_{s, K}$ (see Fig. 3), the excitation in the qubit is swapped into the spin ensemble [9; at a later time it is recovered in the qubit with a fidelity $\sim 0.1$.

A quantum-mechanical description of this experiment 222, 23] is useful in the discussion of the ESR results presented below. Each of the $N_{K}$ effective spins of the ensemble at $\omega_{K}$ is modelled as an effective harmonic oscillator with frequency $\omega_{j_{K}}$ and annihilation (resp. creation) operator $b_{j_{K}}$ (resp. $b_{j_{K}}^{\dagger}$ ), an approximation valid in the low-excitation limit as is the case throughout this article. The spin ensemble and cavity are then described by Hamiltonians $\sum \hbar \omega_{j_{K}} b_{j_{K}}^{\dagger} b_{j_{K}}$ and $\hbar \omega_{c}(\Phi) a^{\dagger} a$, $a$ (resp. $a^{\dagger}$ ) being the cavity annihilation (resp. creation) operator. The coupling between the resonator and the spin ensemble is described by a Hamiltonian $H_{K}=-i \hbar \sum g_{j_{K}}\left(b_{j_{K}}^{\dagger} a+\right.$ h.c. $)$, with $g_{j_{K}}$ the coupling constant of spin $j_{K}$ to the resonator. This Hamiltonian can be rewritten as $H_{K}=-i \hbar g_{K}\left(b_{K}^{\dagger} a+h . c.\right)$ with $g_{K}=\left(\sum g_{j_{K}}^{2}\right)^{1 / 2}$ the spin ensemble-resonator collective coupling constant and $b_{K}=\left(1 / g_{K}\right) \sum g_{\mathrm{j}_{\mathrm{K}}} b_{\mathrm{j}_{\mathrm{K}}}$ the annihilation operator of the collective spin excitation coupled to the cavity. This superradiant mode has a spatial profile given by the spatial dependence of the coefficients $g_{j_{K}}$, which reproduces the profile of the magnetic field inside the cavity mode. Note also that $b_{K}$ involves all the spins belonging to group $K$, even if they have different frequencies due to slightly different magnetic environment in the crystal, also including the three possible states of the ${ }^{14} N$ nuclear spin causing the hyperfine structure; as a result this mode is coupled to $N_{K}-1$ dark modes that act as a bath [16, 22, 24]. Using these notations, one describes the oscillations shown in Fig. 3 as occurring between states $\left|1_{c}, 0_{K}\right\rangle$ and $\left|0_{c}, 1_{K}\right\rangle$, where $\left|1_{c}\right\rangle=a^{\dagger}\left|0_{c}\right\rangle$ is the usual Fock state with 1 photon in the cavity, and $\left|1_{K}\right\rangle=b_{K}^{\dagger}\left|0_{K}\right\rangle$ is the first excited state of the superradiant mode; damping of these oscillations is due to inhomogeneous broadening, and can be interpreted as damping of state $\left|1_{K}\right\rangle$ into the bath of dark states [16].

\section{ESR PROTOCOL AND DISCUSSION}

The ESR protocol is shown in Fig. 4. It consists in an experimental sequence similar to the one used for single photon storage (see fig. 3), but with the spin ensemble initially excited at several photons level instead of the qubit prepared in its excited state. More precisely, a low-power microwave pulse of duration $\Delta t=2 \mu \mathrm{s}$ and varying frequency $\omega_{p}$ is applied to the spins while $\omega_{c}(\Phi)$ is far detuned; the resulting excitation is transferred first into the cavity by tuning $\omega_{c}(\Phi)$ suddenly in resonance with $\omega_{K}$ for the swap time $\tau_{s, K}$, then into the qubit by an adiabatic swap interaction; the qubit state is finally measured. Provided the average number of microwave photons emitted by the spins into the ESR cavity stays much lower than 1 to avoid saturating the qubit, the resulting excited state probability $P_{e}\left(\omega_{p}\right)$ is expected to reproduce the spin ensemble absorption spectrum. Experimental results of Fig. 40 indeed display the characteristic HF structure of $\mathrm{NV}$ centers consisting in three peaks separated by $2.2 \mathrm{MHz}$ for both spin ensembles $+I$ and $+I I I$. This validates the concept of electron spin resonance detected by a superconducting qubit. Note that $P_{e}\left(\omega_{p}\right) \ll 1$ showing that qubit saturation is avoided as wanted.

We now discuss the sensitivity of this qubit-based ESR spectrometer. The qubit state is detected in a single-shot with a fidelity of $\simeq 0.7$ at the end of an experimental sequence that lasts typically $50 \mu \mathrm{s}$, yielding a $1 \%$ precision on the probability $P_{e}$ in one second. To translate this sensitivity in a magnetic moment unit, one needs to know with what efficiency the excitation of the spin ensemble is actually transferred to the qubit. The transfer of one microwave photon from the cavity to the qubit is performed with an efficiency of order unity (in our experiment it is around 0.7 limited by losses in the cavity and qubit), so the limiting factor is the efficiency of the transfer of the spin ensemble excitation to the cavity during their resonant interaction. At first sight one might think that since the spin ensemble and cavity are in the strong coupling limit, one excitation of the spin ensemble should also be converted into a microwave photon with an efficiency of order 1, similar to what happened in the coherent oscillations shown in Fig. 3. This reasoning is not correct here because the collective spin mode $b_{\omega_{p}}$ excited by the spectroscopy pulse does not necessarily match perfectly the superradiant mode $b_{K}$. Indeed, al- 


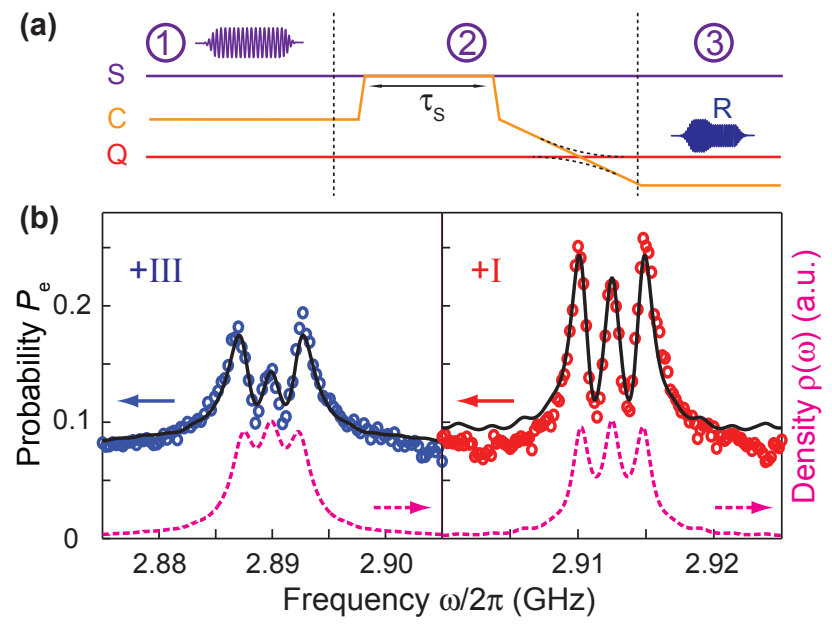

Figure 4. a, Experimental pulse sequence used for qubitdetected ESR: (1) The spins are first weakly excited by a $2 \mu \mathrm{s}$ microwave pulse with a frequency $\omega_{p} ;(2)$ the resulting spin excitation is transferred to the cavity $C$ by a fast flux pulse which brings $\omega_{c}$ in resonance with $\omega_{K}(K=+I,+I I I)$ for a swap time $\tau_{s, K}$, and then to the qubit $Q$ by an adiabatic swap $(a S W A P)$. (3) The qubit excited state probability $P_{e}$ is finally measured. $\mathbf{b}$, Measured (open circles) and calculated (solid line) $P_{e}\left(\omega_{p}\right)$ for spin ensemble $+I I I$ (left) and $+I$ (right). The spin density $\rho(\omega)$ used in the calculation is shown as a dashed line.

though the spatial matching of the two modes is excellent since the specroscopy pulse is applied through the cavity, this is not the case for spectral matching: only spins having a resonance frequency within the spectroscopy pulse bandwidth $\delta / 2 \pi=150 \mathrm{kHz}$ around $\omega_{p}$ contribute to $b_{\omega_{p}}$, whereas all spins within the hyperfine line (total width $\Delta / 2 \pi \sim 5 \mathrm{MHz}$ ) contribute to the superradiant mode $b_{K}$. As a result, one expects an overlap of order $\sqrt{\delta / \Delta}$ between the $b_{\omega_{p}}$ and $b_{K}$ modes, implying that there should be $\sim \Delta / \delta=20$ times less excitations in the $b_{K}$ mode (and thus also in the cavity mode after the swap interaction) than in the $b_{\omega_{p}}$ mode.

This argument can be made rigorous and quantitative. The collective spin mode excited by the spectroscopy pulse is defined as $b_{\omega_{p}}=\left[\sum g_{j_{K}} \alpha_{\omega_{p}}\left(\omega_{j_{K}}\right) b_{j_{K}}\right] / \sqrt{\sum g_{j_{K}}^{2}\left|\alpha_{\omega_{p}}\left(\omega_{j_{K}}\right)\right|^{2}}$, with $\alpha_{\omega_{p}}(\omega)$ the pulse Fourier transform. The quantity of interest is then the correlation function $\beta\left(\omega_{p}, \tau_{s}\right)=\left\langle a\left(\tau_{s}\right) b_{\omega_{p}}^{\dagger}(0)\right\rangle$ giving the probability amplitude for an excitation created in $b_{\omega_{p}}$ by the spectroscopy pulse to be transferred into a photon inside the cavity mode after an interaction time $\tau_{s}$. This function can be computed numerically given a certain spin distribution $\rho(\omega)$ using the formulas derived in the Appendix. In our experiment, the linewidth of each hyperfine peak $w_{+I} / 2 \pi=1.6 \mathrm{MHz}$ and $w_{+I I I} / 2 \pi=2.4 \mathrm{MHz}$, and coupling constants $g_{+I} / 2 \pi=2.9 \mathrm{MHz}$ and $g_{+I I I} / 2 \pi=3.8 \mathrm{MHz}$ have been determined from other measurements [9], so that a direct comparison with theory without any adjustable parameter is possible as shown in Fig. 4. The agreement is quantitative (note that we have also included in the distributions $\rho(\omega)$ additional ESR frequencies caused by the hyperfine interaction of the NV center with neighboring ${ }^{13} C$ nuclei with the $1.1 \%$ natural abundance as expected). From this calculation, we deduce that the average excitation of the spin ensemble at resonance in the data shown in Fig. 4 is $\sim 15$, in agreement with the qualitative argument presented above. In the present state of the experiment, the qubit-based ESR spectrometer therefore measures the spectrum of an ensemble of $10^{11} \mathrm{NV}$ centers at an excitation level of order $15 \mu_{B}$, in a one minute integration time.

Thanks to this very low excitation level, the experimental sequence can be safely repeated at $20 \mathrm{kHz}$, despite the NV centers energy relaxation time reaching minutes at $30 \mathrm{mK}[3,20$. More precisely, two factors contribute to make this experiment possible: 1) at the end of each experimental sequence the excitation of the $b_{\omega_{p}}$ mode quickly decays into the bath of dark modes, allowing the next experimental sequence to start with $b_{\omega_{p}}$ in its ground state and thus keeping the average number of excitations transfered to the qubit well below 1 as needed to avoid saturation, 2) the low excitation rate ensures on the other hand that the ensemble of $10^{11}$ spins stays far from saturation even after repeating the sequence for hours.

We finally note that our calculation reproduces a puzzling feature of the data that was not discussed yet: the middle peak of the $P_{e}\left(\omega_{p}\right)$ curve has a lower amplitude than the two other peaks, both for the $+I$ and the $+I I I$ curves as seen in Fig. 4, although the spin density $\rho(\omega)$ used in the calculation is a simple sum of three Lorentzians with the same amplitude. Our ESR protocol thus appears to slightly distort the absorption spectrum. This phenomenon originates from the $\omega_{p}$ dependence of the energy transfer efficiency from the spins into the cavity, caused by the fact that $g_{K} \approx \Delta$ in our sample. It could probably be corrected in future experiments either by increasing $g_{K}$ or by transferring the spin excitation to the cavity with an adiabatic passage.

Besides detecting a large ensemble of $N=10^{11}$ electronic spins at near single-excitation level, it is interesting to discuss what is the minimal number of spins $N_{\min }$ that could be detected with a similar experimental protocol in order to compare it to the sensitivity of existing conventional spectrometers. For that we will change perspective in the following discussion, and assume that the spins being measured can actually be excited at saturation. We suppose that the $N$ spins, of inhomogeneous linewidth $\Delta$, have been excited by a hard $\pi / 2$ pulse. In the weak coupling limit $g \sqrt{N} \ll \kappa \ll \Delta\left(\kappa=\omega_{c} / Q\right.$ being the cavity damping rate), the spins emit in the cavity $\bar{n}=g^{2} N^{2} /\left(4 \Delta^{2}\right)$ photons (see Appendix B). Taking a conservative estimate for the minimal average excitation that can be detected by a superconducting qubit within one second to be 0.05 , a spin-cavity coupling constant 
$g / 2 \pi=10 \mathrm{~Hz}$, one obtains $N_{\min }=10^{5} \mathrm{spins} / \sqrt{\mathrm{Hz}}$ for a $0.1 \mathrm{mT}$ linewidth corresponding to $\Delta / 2 \pi=2.8 \mathrm{MHz}$. This figure is five orders of magnitude better than a commercial spectrometer at $300 \mathrm{~K}$, and two orders of magnitude better than the record sensitivity of $10^{6}$ spins $/ \sqrt{\mathrm{Hz}}$ for a $0.01 \mathrm{mT}$ linewidth that was recently reported with a surface loop-gap resonator operated at $10 \mathrm{~K} \mathrm{[26]} \mathrm{and} \mathrm{a}$ coplanar waveguide resonator at $4 \mathrm{~K}[5]$. Note however that in order to operate such a qubit-based spectrometer in practice, one would need 1) to use a repetition rate around $10 \mathrm{kHz}$ which requires a reasonably short spinlattive relaxation time or some way to repump rapidly the spins into their ground state (optically [25] or electrically for instance) and 2) to find a qubit design that withstands large magnetic fields usually needed for ESR. Interesting alternative possibilities could be to physically separate the qubit-detector from the spins, which would allow more easily to apply large magnetic fields to the spins without perturbing the qubit, and to continuously monitor the qubit state with a parametric amplifier as demonstrated in recent experiments [27] instead of pulsing the qubit state detection as done here.

In conclusion we have discussed a new type of ESR spectrometer in which the signal coming from the spins is detected by a superconducting qubit acting as a singlemicrowave-photon detector. We have implemented this idea on an ensemble of $\sim 10^{11} \mathrm{NV}$ centers coupled to a transmon qubit, measuring their absorption spectrum at an excitation level of $\sim 15 \mu_{B}$, with a well-resolved hyperfine structure. Estimates indicate that this spectrometer would be able to detect $10^{5}$ spins $/ \sqrt{\mathrm{Hz}}$ with a $0.1 \mathrm{mT}$ linewidth, a gain of two orders of magnitude in sensitivity compared to the best reported values for conventional spectrometers. Our work thus demonstrates the potential of superconducting circuits for electron spin resonance spectroscopy.

\section{APPENDIX A}

We now explain in more detail how the theory curves in figure 3 are calculated. As explained in the main text, the energy transfer efficiency from the resonator to the qubit is of order unity and can be well modeled by an ideal adiabatic passage. In this context the quantity of interest is the resonator population after the interaction with the spins. The calculations are performed in the Holstein-Primakoff approximation, in which the spins and the resonator are described by harmonic oscillators. The total system Hamiltonian is $H / \hbar=\omega_{\mathrm{c}}(\Phi) a^{\dagger} a+\sum \omega_{\mathrm{j}} b_{\mathrm{j}}^{\dagger} b_{\mathrm{j}}+\sum i g_{\mathrm{j}}\left(b_{\mathrm{j}}^{\dagger} a-b_{\mathrm{j}} a^{\dagger}\right), g_{\mathrm{j}}$ being the coupling constant of spin $j$ with the resonator. We need to calculate the probability that the excitation created at $t=0$ in the spins to be transferred to the cavity after a time $t$, this probability is the square modulus of $\left\langle 0\left|a(t) b_{\omega_{p}}^{\dagger}\right| 0\right\rangle$. The spins excitation is created by a microwave pulse of central frequency $\omega_{p}$ with a pulse envelope in frequency described by $\alpha_{\omega_{p}}(\omega)=\alpha\left(\omega-\omega_{p}\right)$, a typical envelope is a Lorentzian function with FWHM $\delta$. We can define an operator $b_{\omega_{p}}^{\dagger}$ that describes the excitation induced by this pulse as

$$
b_{\omega_{p}}^{\dagger}=\frac{1}{\sqrt{\sum_{j}\left|\alpha_{\omega_{p}}\left(\omega_{j}\right)\right|^{2} g_{j}^{2}}} \sum_{k} \alpha_{\omega_{p}}\left(\omega_{k}\right) g_{k} b_{k}^{\dagger},
$$

this comes simply from the standard atom-field interaction for a classical light source such as the one used in the experiment. As shown in [22] the quantity $\left\langle 0\left|a(t) b_{\omega_{p}}^{\dagger}\right| 0\right\rangle$ can be calculated by considering an effective non-Hermitian Hamiltonian

$$
H_{e f f} / \hbar=\left(\begin{array}{cccc}
\tilde{\omega}_{0} & i g_{1} & i g_{2} & \ldots \\
-i g_{1} & \tilde{\omega}_{1} & & \\
-i g_{2} & & \tilde{\omega}_{2} & \\
\vdots & & & \ddots
\end{array}\right)
$$

with complex angular frequencies $\tilde{\omega}_{0}=\omega_{c}(\Phi)-i \kappa / 2$ and $\tilde{\omega}_{k}=\omega_{k}-i \gamma_{0} / 2$; here, $\gamma_{0}$ is the spontaneous emission rate of each spin (that we take here to be zero since NV centers at low temperature have negligible energy relaxation). Indeed, introducing the vector $X(t)$ of coordinates $\left[\left\langle a(t) a^{\dagger}(0)\right\rangle, \ldots,\left\langle b_{j}(t) a^{\dagger}(0)\right\rangle, \ldots\right]$ it can be shown that $d X / d t=-(i / \hbar) H_{\text {eff }} X$. The formal solution to this equation is then

$$
X(t)=\mathcal{L}^{-1}\left[\left(s+i H_{e f f} / \hbar\right)^{-1} X(0)\right],
$$

which gives $\left\langle 0\left|a(t) b_{\omega_{p}}^{\dagger}\right| 0\right\rangle=x_{G}^{\dagger} \cdot X(t)=\mathcal{L}^{-1}\left[t_{\omega_{p}}(s)\right]$ with $x_{G}=(1,0,0, \ldots)$ and $\mathcal{L}[f(s)]=\int e^{-s t} f(t) d t(s$ being a complex number). The initial condition $X(0)$ is the one produced by $b_{\omega_{p}}^{\dagger}$ given in Eq,1, thus

$$
\begin{aligned}
t_{\omega_{p}}(-i \omega) & =\frac{\sum_{k} \alpha_{\omega_{p}}\left(\omega_{k}\right) g_{k}}{\sqrt{\sum_{j}\left|\alpha_{\omega_{p}}\left(\omega_{j}\right)\right|^{2} g_{j}^{2}}}\left[\left(s+i H_{e f f}\right)^{-1}\right]_{0, k} \\
& =\frac{\sum_{k} \alpha_{\omega_{p}}\left(\omega_{k}\right) g_{k}}{\sqrt{\sum_{j}\left|\alpha_{\omega_{p}}\left(\omega_{j}\right)\right|^{2} g_{j}^{2}}}\left[\frac{g_{k} t_{1}(-i \omega)}{i \gamma_{0}+\left(\omega-\omega_{p}\right)}\right] \\
& =\frac{t_{1}(-i \omega)}{i \gamma_{0}+\left(\omega-\omega_{p}\right)} \frac{\sum_{k} \alpha_{\omega_{p}}\left(\omega_{k}\right) g_{k}^{2}}{\sqrt{\sum_{j}\left|\alpha_{\omega_{p}}\left(\omega_{j}\right)\right|^{2} g_{j}^{2}}},
\end{aligned}
$$

where $t_{1}(-i \omega)=i /\left[\omega-\omega_{0}+i \kappa / 2-W(\omega)\right]$ with $W(\omega)=\sum_{j} g_{j}^{2} /\left[\omega-\omega_{j}+i \gamma_{0} / 2\right]$. Note that we evaluated $t_{\omega_{p}}(s)$ for $s=-i \omega$, this is sufficient to perform the Laplace transform inversion as there are no singularities in the imaginary axis of $t_{\omega_{p}}$. We define the spin density $\rho(\omega)$ encompassing the coupling strength, which is possibly different for each spin, as $\rho(\omega)=\sum_{j} \frac{g_{j}^{2}}{g_{K}^{2}} \delta\left(\omega-\omega_{j}\right)$. Using this definition in the equation above we have

$$
t_{\omega_{p}}=\frac{g_{K} t_{1}(-i \omega)}{i \gamma_{0}+\left(\omega-\omega_{p}\right)} \frac{(\alpha * \rho)\left(\omega_{p}\right)}{\sqrt{\left(|\alpha|^{2} * \rho\right)\left(\omega_{p}\right)}} .
$$


The spectral width of the microwave pulse is, in our case, much smaller than any scale that characterizes our distribution $\rho(\omega)$. This allows the rewriting of the convolution above as

$$
\frac{(\alpha * \rho)\left(\omega_{p}\right)}{\sqrt{\left(|\alpha|^{2} * \rho\right)\left(\omega_{p}\right)}}=A \sqrt{\rho\left(\omega_{p}\right)}
$$

where the constant $A=\frac{\int \alpha(\omega) d \omega}{\sqrt{\int|\alpha(\omega)|^{2} d \omega}}$ is purely characterized by the pulse envelope with no dependence on $\omega_{p}$, yielding for example $A=\sqrt{\delta} \sqrt{\pi / 2}$ for a Lorentzian envelope. This means that if we consider that the spins are distributed at a typical range $\Delta$ the equation above gives a rigorous justification of the rule of thumb that says that the efficiency of the spins-resonator transfer is given by the overlap $\sqrt{\delta / \Delta}$.

Finally to generate the theoretical curve in Fig. 3, we perform a numerical inversion of the Laplace transform for each $\omega_{p}$ and take $\left|\left\langle 0\left|a(t) b_{\omega_{p}}^{\dagger}\right| 0\right\rangle\right|^{2}$ at $t=\tau_{S, I I I}$ or $t=\tau_{S, I}$.

\section{APPENDIX B}

We now explicit the calculation of the sensitivity of our qubit-based ESR spectrometer in the weak coupling limit $g_{K} \ll \kappa \ll \Delta$. The Hamiltonian coupling the spins to the cavity field is $H=\hbar g\left(S_{-} a^{\dagger}+h . c.\right)$, where $S_{-}=\sum \sigma_{i,-}, \sigma_{i,-}$ being the lowering operator of spin $i$. In the absence of driving field, the equation for the intra-cavity mean field is then easily obtained as

$$
\frac{d<a>}{d t}=-\frac{\kappa}{2}<a>-i g<S_{-}>
$$

Right after a $\pi / 2$ pulse on the spins, $\left|\left\langle S_{-}\right\rangle\right|=N / 2$. Neglecting the back-action of the cavity field on the spins (which is justified in the weak coupling limit), we simply get that $\left\langle S_{-}\right\rangle=(N / 2) e^{-\Delta t}$. From that one shows that $\langle a\rangle(t)=-i g N\left(e^{-\kappa t / 2}-e^{-\Delta t}\right) /[\kappa-2 \Delta]$ which in the limit $\kappa \ll \Delta$ yields a maximum photon number in the cavity of $\bar{n}=g^{2} N^{2} /\left(4 \Delta^{2}\right)$.

Acknowledgements We acknowledge useful discussions with B. Julsgaard, K. Moelmer, J. Morton, A. Briggs, and within the Quantronics group, and technical support from P. Sénat, P.-F. Orfila, T. David, J.-C. Tack, P. Pari, P. Forget, M. de Combarieu. We acknowledge support from European project Solid, ANR projects Masquelspec and QINVC, C'Nano, Capes, and Fondation Nanosciences de Grenoble.
[1] Y. Kubo et al., Phys. Rev. Lett. 105, 140502 (2010).

[2] D.I. Schuster, et al., Phys. Rev. Lett. 105, 140501 (2010).

[3] R. Amsuss, et al., Phys. Rev. Lett. 107, 060502 (2011).

[4] P. Bushev et al., Phys. Rev. B 84, 060501 (2011).

[5] H. Malissa, D. I. Schuster, A.M. Tyryshkin, A.A. Houck, and S.A. Lyon, arxiv:1202.6305.

[6] Y.-F. Chen et al., Phys. Rev. Lett. 107, 217401 (2011).

[7] G. Wendin and V.S. Shumeiko, Low Temperature Physics 9, 724 (2007).

[8] Note that in a real ESR spectrometer, the FID signal would in fact be difficult to detect using this protocol because of ringing of the cavity and of the detectors dead-time. The spin absorption spectrum would therefore rather be measured by CW-ESR rather than by this FIDdetected protocol. We nevertheless discuss it for pedagogical reasons, because it is closest to our qubit-detected ESR experiment in which these technical issues are irrelevant.

[9] Y. Kubo et al., Phys. Rev. Lett. 107, 220501 (2011).

[10] F. Jelezko, T. Gaebel, I. Popa, A. Gruber, and J. Wrachtrup, Phys. Rev. Lett. 92, 076401 (2004).

[11] S. Felton, et al., Phys. Rev. B 79, 075203 (2009).

[12] The experiment was performed in the same magnetic field as in [9] where the reported value was mistakenly mentioned to be $B_{N V}=1.4 \mathrm{mT}$.

[13] L. Frunzio, A. Wallraff, D. I. Schuster, J. Majer, and R. J. Schoelkopf, IEEE Trans. on Applied Superconductivity 15, 860 (2005).

[14] A. Palacios-Laloy et al., J. Low Temp. Phys. 151, 1034 (2008).

[15] M. Sandberg, et al., Appl. Phys. Lett. 92, 203501 (2008).

[16] Y. Kubo, et al., Phys. Rev. A 85, 012333 (2012).

[17] J. Koch et al., Phys. Rev. A 76, 042319 (2007).

[18] J. A. Schreier et al., Phys. Rev. B 77, 180502 (2008).

[19] F. Mallet, et al., Nature Phys. 5, 791 (2009).

[20] A. Jarmola, V. M. Acosta, K. Jensen, S. Chemerisov, D. Budker, arxiv:1112.5936

[21] X. Zhu et al., Nature 478, 221 (2011).

[22] I. Diniz et al., Phys. Rev. A 84, 063810 (2011).

[23] Z. Kurucz, J.H. Wesenberg and K. Molmer, Phys. Rev. A 83, 053852 (2011).

[24] K. Sandner et al., arxiv:1112.4767 (2011).

[25] A. Yang et al., PRL 102, 257401 (2009).

[26] Y. Twig, E. Dikarov, W.D. Hutchison, and A. Blank, Rev. of Sci. Instr. 82, 076105 (2011).

[27] R. Vijay et al., Phys. Rev. Lett. 106, 110502 (2011). 